\title{
A Pharmacist-Led Medication Use Evaluation of
}

\section{Proton-Pump Inhibitors}

\author{
Kathryn L. Velasquez ${ }^{1}$, An M. Alvarez ${ }^{1}$ and Portia N. Davis ${ }^{1,2}$ \\ 1. College of Pharmacy and Health Sciences, Texas Southern University, Houston 77004, Texas, USA \\ 2. San José Clinic, Houston 77002, Texas, USA
}

\begin{abstract}
Objective: To determine whether patients maintain symptom control after discontinuation of PPI (proton-pump inhibitor) therapy through intervention of a pharmacist-led medication utilization program and determine annual clinic cost-savings per prescription. Design: The study was conducted as a prospective, medication use evaluation. Using an electronic medical record system at a clinic, reports generated all patients receiving PPI therapy from January 2013-June 2015. Patients were encouraged to participate in a PPI discontinuation trial through a pharmacist-led educational session. Participants were followed-up three months post enrollment to assess their quality of life through a survey assessing severity and frequency of symptoms. Participants unable to maintain symptom control after the intervention were referred back to their primary care physician for further evaluation. Results: Of one hundred participants, $25 \%(n=25)$ were able to discontinue the use of PPI by lifestyle modifications, $43 \%(n=43)$ refused to discontinue PPI therapy and lifestyle changes due to the severity of their symptoms, $17 \%(n=17)$ switched to over-the-counter $\mathrm{H}_{2}$ receptor antagonist daily to control their symptoms, $9 \%(n=9)$ used PPIs only as needed, and $6 \%(n=6)$ of participants were dropped from the study after three failed communication attempts. The clinic saved approximately \$11 thousand annually for every one prescription of PPI's. Conclusions: Discontinuation of PPI or step-down therapy was possible for patients with mild-moderate GERD (Gastroesophageal Reflux Disease) symptoms when mediated by pharmacist counseling and follow-up. Also, the annual PPI expenditure at this clinic will decrease due to participant's discontinuation of therapy.
\end{abstract}

Key words: Discontinuation, proton-pump inhibitors, overutilization, GERD, cost-savings.

\section{Introduction}

PPIs (Proton pump inhibitors) are very effective agents in suppressing stomach acid production. They are indicated for the treatment of GERD (Gastroesophageal Reflux Disease), peptic ulcer disease, NSAID-induced ulcer prevention, Zollinger-Ellinson syndrome, erosive esophagitis, Barret's esophagus and upper gastrointestinal hemorrhage[1,2]. The Guidelines in the Diagnosis and Management of GERD recommend the use of PPIs for a period of 8 weeks. Maintenance treatment is reserved to those that continue to be symptomatic after PPI discontinuation $[1,2]$.

GERD is usually not a life-threatening disease; however, symptoms have been known to affect patients'

Corresponding author: Portia N. Davis, Pharm.D., RPh., research fields: pharmacy and pharmaceutical care. quality of life. Approximately 40 percent of the adult American population is affected by GERD monthly [1]. The increasing prevalence of GERD has caused a significant rise in the use of PPIs. PPIs are currently the first-line agent in treating GERD and account for a cost of approximately $\$ 11$ billion annually in the USA. In 2015 the PPI Esomeprazole (Nexium ${ }^{\circledR}$ ) was listed in the top ten highest drug sales of America with 13.2 million prescriptions sold totaling $\$ 4.7$ billion [3]. Such high volume of sales has warranted researchers to further explore the necessity of PPI prescribing. Mostly due in part to its severe potential risk associated with the long-term use of this medication class. Clostridium difficile, associated diarrhea and other gastrointestinal infection along with increased risk of osteoporosis-related bone fractures are just a few of the risk associated with prolong use of PPI therapy [4].

Recent studies have also discovered PPI use to be 
associated with an increased risk of AKI (acute kidney injury), incident CKD (chronic kidney disease), and progression to ESRD (end-stage renal disease) [5].

Due to severe potential risk side effects such as these, it is imperative for practitioners to set in place an MUE (medication use evaluation) program to prevent overutilization of prescription drugs. MUE programs not only aid healthcare facilities in a variety of ways but are mandated by the Omnibus Budget Reconciliation Act of 1990 for all Medicaid participants [6]. Oftentimes physicians prescribe PPIs intended for a short therapy course, however, due to patient or physician failure to follow-up, these short course therapies result in long-term use. Because of the risk factors associated with the prolong use of PPIs, it is important that patients follow-up with their physicians for reevaluation. Studies have revealed that overutilization of PPIs happens quite frequently and occurs primarily in patients requiring long-term treatment [7].

There are several PPI discontinuation interventional programs described in literature. A Veterans Administration program was designed to assess the feasibility of step-down therapy on outpatients with GERD. Seventy-three patients that had been on PPI therapy for at least 8 weeks and did not have GERD symptoms, were included. Furthermore, $58 \%$ of patients were successfully discontinued from PPI treatment after one year of follow-up and 34\% required an H2-blocker. Quality of life was not affected by discontinuation, and treatment costs were decreased by $37 \%$ [8].

This study focuses on a pharmacist-led, medication-use evaluation for patients receiving long-term PPI therapy. Through pharmacist-led intervention, participants were able to de-escalate PPI therapy resulting in the elimination of potential risk factors associated with prolong use and decrease their daily medication intake burden. The intervention also aided in minimizing clinic expenditures.

\section{Methods}

This study was designed as a prospective, medication use evaluation and was conducted at a charity clinic in Houston, Texas. SJC (San Jose Clinic) is a non-profit clinic which provides health care services, including medications, to uninsured low-income patients.

Data was collected and recorded without patient identifiers to ensure patient confidentiality. Reports were generated through the electronic medical record system, Sevocity, identifying all patients receiving PPI therapy from January 2013 to June 2015. From May to September 2016, participants 18 years of age and older were contacted twice via telephone. The initial outreach engaged patients in a pharmacist-led counseling session which encouraged them to participate in a PPI discontinuation trial. During the counseling session, participants were asked to consider step-down therapy which included lifestyle modifications and OTC $\mathrm{H}_{2} \mathrm{RA}$ (over-the-counter $\mathrm{H}_{2}$ receptor antagonist) to aid with GERD symptoms throughout the study. Three months following initial outreach, patients received a second call to evaluate their step-down therapy approach. A survey was also conducted at this time to assess their GERD symptoms to date $[9,10]$.

An estimated annual cost-saving per prescription was calculated based on a once daily dosing regimen. The average wholesale cost per capsule was multiplied by thirty which indicated the monthly cost and then multiplied once again by twelve to derive the annual cost-savings per prescription.

\section{Results}

A total of one hundred patients receiving PPI therapy were attempted to be reached. Six percent $(n=6)$ of patients were dropped from the study after three failed attempts to reach. Of those remaining, $25 \%(n=25)$, between the ages of 18 to 49 , were successfully able to discontinue PPI therapy without the necessity of an 
OTC $\mathrm{H}_{2} \mathrm{RA}$. These patients managed to maintain symptom control through lifestyle modifications involving diet adjustments. A total of 43\% $(n=43)$ refused to discontinue PPI therapy or consider any lifestyle modifications due to the severity of their symptoms. Patients that were able to adhere to step-down therapy using OTC $\mathrm{H}_{2} \mathrm{RA}$ daily totaled $17 \%$ $(\mathrm{n}=17)$ and those still requiring PPI therapy as needed for symptom control totaled nine percent $(n=9)$ (Fig. 1).

An annual cost per Esomeprazole prescription is approximately \$3 thousand, \$2 thousand for Omeprazole, \$2 thousand for Pantoprazole, and \$4 thousand for Rabeprazole (Table 1, Fig. 2).

\section{Discussion}

As previously outlined, overutilization of PPIs occurs quite frequently. The most common indications in the study were GERD, $\mathrm{H}$ pylori infection and gastritis. In some cases, there was no indication. Although NSAID-induced ulcer prophylaxis is an accepted indication for PPI treatment, no patients were prescribed PPIs for this indication. Patients on glucocorticoid therapy, history of GI ulcers and stomach cancer, and those at high risk of bleeding such as NSAID + clopidogrel treatment were excluded from the intervention. A patient with CKD was also excluded as well as a patient scheduled for an endoscopy.

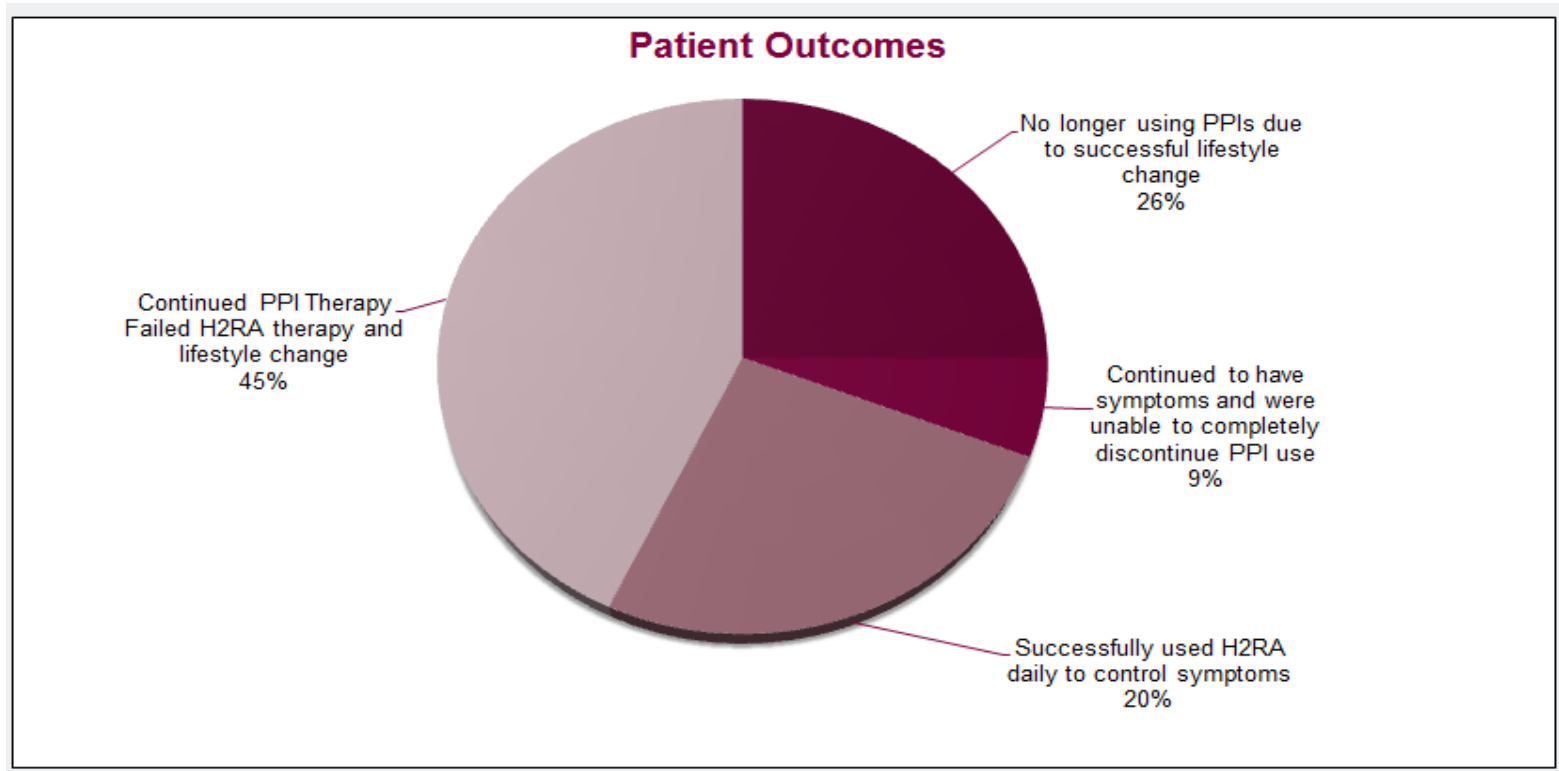

Fig. 1 Distribution of total 96 participants.

Table 1 PPI prescriptions from 2010-2014 at SJC including average cost per unit.

\begin{tabular}{lll}
\hline PPI & No. prescriptions & AWP/unit \\
\hline Esomeprazole $20 \mathrm{mg}$ & 29 & $\$ 8.53 /$ capsule \\
Esomeprazole $40 \mathrm{mg}$ & 487 & $\$ 8.53 /$ capsule \\
Omeprazole $20 \mathrm{mg}$ & 181 & $\$ 4.45 /$ capsule \\
Pantoprazole $20 \mathrm{mg}$ & 16 & $\$ 5.27 /$ tablet \\
Pantoprazole $40 \mathrm{mg}$ & 270 & $\$ 5.27 /$ tablet \\
Rabeprazole $20 \mathrm{mg}$ & 50 & $\$ 11.44 /$ capsule \\
Total prescriptions & 1,033 & \\
\hline
\end{tabular}

SJC: San José Clinic, AWP: average wholesale price.

Note. The listed PPIs reflect the currently approved PPIs on the SJC formulary. 


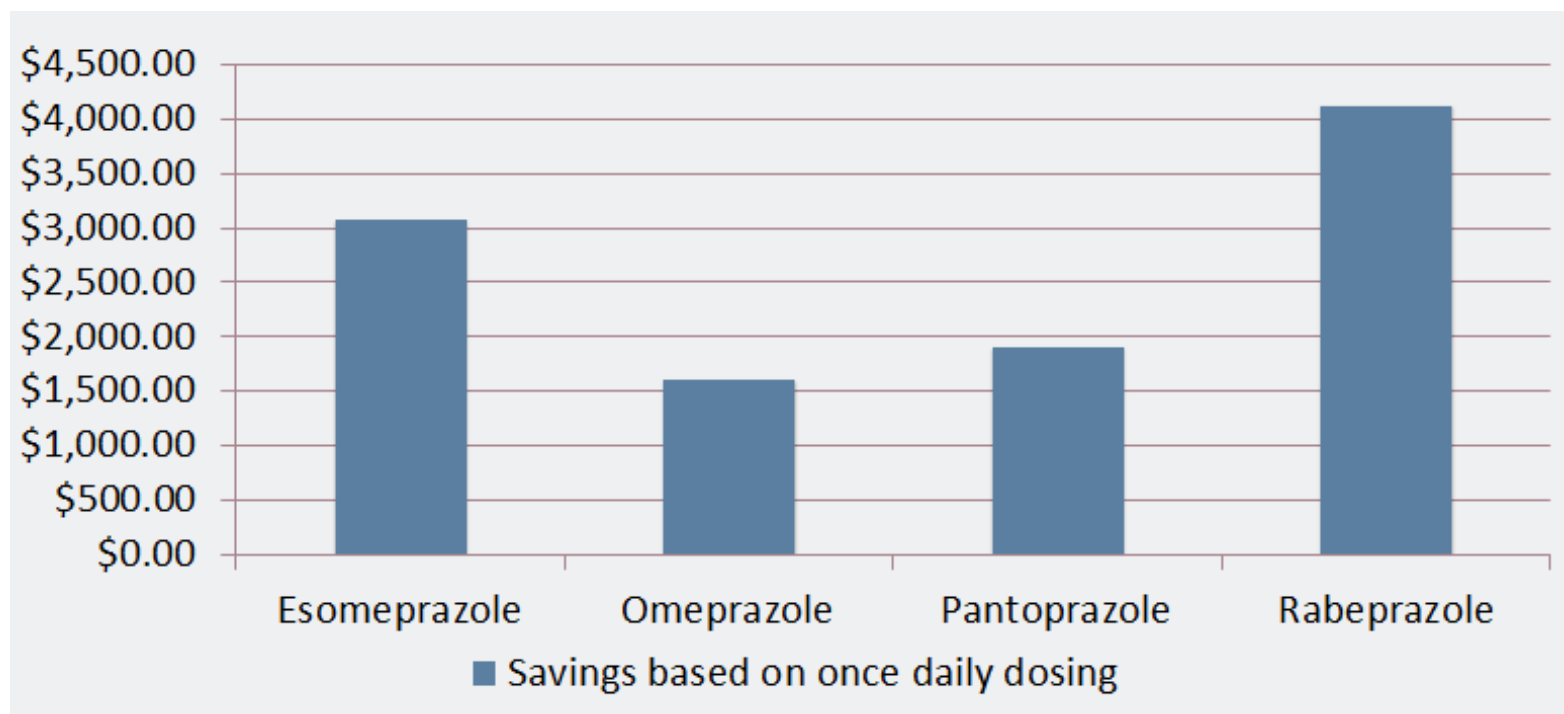

Fig. 2 Estimated annual clinic savings per prescription from proper discontinuation.

It is important to adhere to MUE programs so that the opportunity to de-escalate therapy is not missed. De-escalation of PPI therapy eliminates the risk associated with prolong use of treatment and decreases the daily medication intake burden. Unfortunately, it is merely impossible to follow-up with every patient. As illustrated in this study, six of one hundred patients were unable to participate due to three failed attempts to reach. Patients that agreed to discontinue PPI use were generally 49 years or younger and took fewer maintenance medications. Though not every attempt was successful, forty-two participants were able to de-escalate therapy solely through pharmacist-led intervention resulting in significant clinic cost-savings. The information gathered in this study further reveals the importance of conducting periodic MUEs. It is required by law for all Medicaid recipients to be provided with a periodic MUE, and it is an excellent method to control medication cost and overutilization.

Patient's benefits included: improved quality of life, decreased pill burden, lower risk for adverse drug events, and decreased health cost. Longer follow-up times will be evaluated in the future to assess long-term success of the intervention.

\section{Conclusions}

The desired intervention of the pharmacist-led
MUE is to educate physicians and patients of the potential risk associated with prolonged and inappropriate PPI use. Patient hesitation to discontinue PPI therapy is an expected barrier because patients may experience rebound symptoms such as heartburn, indigestion, or acid regurgitation. Studies have revealed that the main reason for reuptake of therapy is due to rapid recurrence of gastric reflux symptoms [11].

However, educating the patient on lifestyle modifications and alternative therapies will serve to control recurring symptoms.

Discontinuing patients from PPI therapy or step-down therapy with OTC $\mathrm{H}_{2}$-blockers is feasible for patients with mild-moderate GERD symptoms upon PPI discontinuation. Furthermore, discontinuation of inappropriate PPI use will also decrease clinic expenditures. Such as, discontinuation of just one prescription from the aforementioned PPIs resulted in an annual cost-savings of $\$ 11$ thousand. As a result, longer follow-up times may be required to fully evaluate the success of the intervention.

\section{Acknowledgments}

This study was supported by the collaboration of Texas Southern University College of Pharmacy and Health Sciences and the San José Clinic. We thank San 
José Clinic for allowing us access to their database to conduct this research, and Mr. Carlos Velasquez for his visual representation in the studies poster presentation. We would also like to express our gratitude to Dr. Portia N. Davis, Assistant Professor of Pharmacy Practice at Texas Southern University College of Pharmacy and Health Sciences for her insight and comments that improved the studies poster presentation and manuscript.

\section{References}

[1] Katz, K. O., Gerson, L. B., and Vela, M. F. 2013. "Corrigendum: Guidelines for the Diagnosis and Management of Gastroesophageal Reflux Disease.” The American Journal of Gastroenterology 108: 308-28. doi: 10.1038/ajg.2012.444

[2] Katz, P., Gerson, L., and Vela, M. 2013. "Diagnosis and Management of Gastroesophageal Reflux Disease.” Am J Gastroenterol 108: 308-28.

[3] Brooks, M. 2014. "Top 100 Most Prescribed, Top Selling Drugs.” Medscape Medical News. Retrieved from http://www.medscape.com/viewarticle/825053.

[4] Esomeprazole. Lexi-Drugs. Lexicomp. Wolters Kluwer Health, Inc. Hudson, OH. Accessed on February 20, 2017. Available at: http://online.lexi.com.

[5] Xie, Y., Bowe, B., Li, T. T., Xian, H., Yan, Y., and Al-Aly, Z. (2017). "Long-Term Kidney Outcomes among Users of Proton Pump Inhibitors without Intervening Acute Kidney
Injury.” Kidney International 91 (6): 1482-94. doi: http://dx.doi.org/10.1016/j.kint.2016.12.021

[6] U.S. Congress. H.R. 5385, Omnibus Budget Reconciliation Act of 1990, Public Law 101-508, 101st Congress (November 5, 2009). [Section 1927, g. 2 A, B]. Accessed March 1, 2017. Available from: https://www.congress.gov/bill/101st-congress/house-bill/ 5835.

[7] Heidelbaugh, J. J., Andrea, H. K., and Walker, P. C. 2012. "Overutilization of Proton-Pump Inhibitors: What the Clinician Needs To Know.” Therapeutic Advances in Gastroenterology 5:219-32.doi:10.1177/1756283X12437358

[8] Inadomi, J., et al. 2001. "Step-Down Management of Gastroesophageal Reflux Disease.” Gastroenterology 121: 1095-100.

[9] Loge, D., et al. 2004. "Cross-Cultural Development and Validation of a Patient Self-administered Questionnaire to Assess Quality of Life in Upper Gastrointestinal Disorders: The PAGI-QOL.” Quality of Life Research 13: 1751-62.

[10] Macran, S., Wilenma, S., Barton, G., and Rusell, I. 2007. "The Development of a New Measure of Quality of Life in the Management of Gastro-Oesophageal Reflux Disease: The Reflux Questionnaire.” Quality of Life Research 16: 331-43.

[11] Reimer, C., and Bytzer, P. 2010. "Discontinuation of Long-Term Proton Pump Inhibitor Therapy in Primary Care Patients: A Randomized Placebo-Controlled Trial in Patients with Symptom Relapse.” Gastroenterology \& Hepatology 22: 1182-8. 